\title{
sciendo
}

\section{Virtual Realism: Really Realism or only Virtually so? A Comment on D. J. Chalmers's Petrus Hispanus Lectures}

\author{
Claus Beisbart \\ University of Bern \\ DOI: $10.2478 /$ disp-2019-0008 \\ BIBLID [0873-626X (2019) 55; pp.297-331]
}

\begin{abstract}
What is the status of a cat in a virtual reality environment? Is it a real object? Or part of a fiction? Virtual realism, as defended by D. J. Chalmers, takes it to be a virtual object that really exists, that has properties and is involved in real events. His preferred specification of virtual realism identifies the cat with a digital object. The project of this paper is to use a comparison between virtual reality environments and scientific computer simulations to critically engage with Chalmers's position. I first argue that, if it is sound, his virtual realism should also be applied to objects that figure in scientific computer simulations, e.g. to simulated galaxies. This leads to a slippery slope because it implies an unreasonable proliferation of digital objects. A philosophical analysis of scientific computer simulations suggests an alternative picture: The cat and the galaxies are parts of fictional models for which the computer provides model descriptions. This result motivates a deeper analysis of the way in which Chalmers builds up his realism. I argue that he buys realism too cheap. For instance, he does not really specify what virtual objects are supposed to be. As a result, rhetoric aside, his virtual realism isn't far from a sort of fictionalism.
\end{abstract}

\section{Keywords}

Computer simulation, model, fictional model, ontology, fictionalism.

\section{Introduction}

Suppose that ten-year old Tina has asked her parents for a cat as a Christmas present. At Christmas, the parents give her a headset with 
a stereoscopic display and a device. The headset is connected to a computer that is programmed to produce visual impressions of a dining room. In this room, a cat moves around. Using the little device, Tina can move around in the dining room too, which is to say that the perspective from which the room appears changes in a systematic way depending on Tina's input on the device. If Tina sees the cat close nearby, she can use a switch on the device to pet the cat. As a reaction, the cat will typically purr. Suppose for the sake of argument that the cat in the dining room does everything a cat typically does.

Many people would think that this present is a cheat. They would understand if Tina is deeply disappointed. They would not just take it that Tina hasn't been given what she had asked for. Rather, they would think that Tina has just been given a headset with a device and no cat-like object whatsoever. If there was any talk about a cat, it would be about mere fiction, or so they would think.

David J. Chalmers disagrees. On his view, Tina has been given a virtual cat. According to what he endorses under the label "virtual realism", the virtual cat is a real object. It can have properties, e.g. be gray, and be involved in events, e.g. jump on a chair. It can further figure in non-illusory perceptual experience. Chalmers brilliantly defends this position in his Petrus Hispanus Lectures (Chalmers 2017). But isn't the position a sort of cheat too? Is realism really vindicated for virtual reality (VR, for short)?

The aim of this paper is to critically discuss Chalmers's virtual realism and the arguments he puts forward in favor of his position. I focus on Chalmers's preferred specification of virtual realism, which includes what he calls virtual digitalism. I'll begin by briefly recapping Chalmers's virtual realism in Section 2. Section 3 compares virtual reality environments with scientific computer simulations. I argue that, if it is sound, Chalmers's view can, and should, be extended to all sorts of scientific computer simulations. I suggest that this is a slippery slope because it leads to implausible consequences. Section 4 steps back and asks what went wrong in the construction of virtual realism. I identify several problems and argue that Chalmers establishes realism too cheap since fictionalism is just around the corner. This is not to deny that Chalmers's virtual realism raises a number of intriguing issues. I draw my conclusions in Section 5. 


\section{Virtual realism à la Chalmers}

Back to Tina's cat. What does it mean to say that it is a real object? And what does Chalmers's virtual realism imply for the cat?

By construction, the cat "lives" in a dining room. The latter is an example of what Chalmers calls a virtual (reality) environment (2017: 312). ${ }^{1}$ The environment has three characteristic properties, viz. of being immersive, interactive and computer-generated (2017: 312-3). A virtual environment is immersive since it produces perceptions of an environment from a view within it. When Tina wears the headset and the computer program is running properly, she has experiences of a dining room as seen from a certain position and angle. A virtual environment is interactive because the human user can influence the environment. As far as Tina is concerned, she can use the device to move around and to pet the cat, which causes the virtual cat purring. Finally, a virtual environment is computer-generated, no doubts about this in our example.

Chalmers would call the dining room not just a virtual environment, but also a virtual world (2017: 314). The cat is part of this virtual world or an object in the virtual world and for this reason called a virtual object (ibid.).

When developing virtual realism, Chalmers adds to a venerable tradition of philosophical thought. Indeed, in the view of Wright (1993: 1), the debate about realism is constitutive of Western philosophy. As Wright further notes (1993: 2-3), realism is now often discussed with respect to a specific domain of discourse. Here, we are obviously concerned with talk about virtual reality environments. As is typical of every variety of realism, Chalmers's virtual realism is a package of several commitments, some of which are metaphysical, while others are epistemological. Let's consider each component of the package in turn by contemplating Tina's cat.

The metaphysical commitment is that Tina's cat really exists, that it really has properties and that it is involved in events that really take place. This isn't too informative unless we know what the cat is and what it means to say that it has properties etc. Now Chalmers does address these questions very explicitly, and at this point his

\footnotetext{
${ }^{1}$ References are to Chalmers 2017, unless specified otherwise.
} 
virtual digitalism comes into play (2017: 311). So throughout my paper, Chalmers's virtual digitalist specification of virtual realism will be my focus.

Virtual digitalism takes the cat to be a digital object (2017: 317). Such an object is constituted by computational processes in the computer hardware that produce a virtual environment. Chalmers adds that, to a first approximation, the cat can be regarded as a data structure, which in turn is grounded in computations in the hardware. He doesn't consider more complicated settings in which a virtual object is realized using several data structures, and so won't I. As far as properties are concerned, virtual realists hold that the cat qua digital object instantiates virtual properties (2017: 320-1). For Chalmers's virtual digitalist brand of virtual realism, these are digital properties. For instance, the virtual, and thus digital, greyness of the cat comprises those aspects of the digital object (i.e., roughly, of the data structure) that produce the grayish appearance of the cat in the headset. Chalmers thinks that this account can be extended to other virtual/digital properties. In particular, at least some virtual properties are characterized by their roles in virtual reality (2017: 323-4). A similar story is told about virtual, or digital, events. In general terms, then, the metaphysical commitments of Chalmers's virtual realism are as follows (2017: 311):

VR-M1 Virtual objects really exist and are digital objects;

VR-M2 Events in virtual worlds are largely digital events that really take place;

Here, VR-M2 does not explicitly talk about the instantiation of properties, but for the purposes of this paper, the latter may be considered as a special sort of event. For reasons of simplicity, I will focus on the instantiation of properties in what follows. For the time being, we can ignore the qualification "largely" in VR-M2.

In our example with the cat, the epistemological commitment of virtual realism holds that Tina has, or may at least have, non-illusory perception of the cat (2017: 326-33). Tina can thus know about the cat by observing it. She will only have non-illusory perceptions of the cat, of course, if she doesn't take the cat to be a real cat because she would then be deceived. But Chalmers argues that experienced users 
of virtual reality environments form a conception of virtual reality. Their belief that they are concerned with a virtual world then acts as a theoretical assumption that informs the content of their perceptions. Thus, if Tina is an experienced user of her virtual reality device, the content of her perceptual states is e.g. that the virtual cat instantiates the virtual property of being gray. Chalmers masterfully develops this part of his view by comparing with the perception of mirror images.- In general terms, the epistemological commitment of virtual realism reads (2017: 311):

VR-E Experiences in virtual reality involve non-illusory perception of a digital world;

Chalmers's virtual realism finally has an evaluative component (2017: 337-44). In our example, it boils down to the claim that there is no aspect in which Tina's interaction with the virtual cat is significantly less valuable than the interaction with a real cat. (Tina's parents were very happy when they were told this by the seller in the department store in which they bought the virtual reality device.) One crucial thought here is that Tina isn't really subject to illusions, when she plays with the virtual cat (2017: 339). Another argument is that, unlike in Nozick's experience machine, Tina isn't degraded to a passive recipient of all sorts of influences from the computer. Rather, her decisions have an impact on what's going on in the virtual dining room (2017: 339-40). ${ }^{2}$ In general terms, Chalmers's evaluative claim is (2017: 311):

VR-V Virtual experiences of a digital world can be about as valuable as non-virtual experiences of a non-digital world.

Since such an evaluation is not typical for other domain-specific realisms, I won't discuss it here.

For the purposes of the following, it is useful to briefly recap the arguments that Chalmers offers for his position.

The argument from causal power supports the identification of virtual objects with digital objects. The first premise is that virtual objects

${ }^{2}$ Of course, interaction is not specific to VR environments, but rather a feature of so-called interactive media, more generally, e.g. interactive novels (Chalmers 2017: 348). See Lopes (2001), Smuts (2009) and Wildman and Woodward (2018) for accounts of interactive media and the relevant type of interaction. 
can cause all sorts of things (e.g. virtual events, but also experiences in the user). Since it is really digital objects that cause these sorts of things, virtual objects must be digital objects. Put this way, the argument presupposes that there are virtual objects with causal powers. But Chalmers thinks that the argument has even some pull if we only admit that there seem to be objects with causal powers (2017: 318).

The argument from perception too is supposed to support the metaphysical claim that virtual objects are digital objects. The argument assumes that we perceive virtual objects. It is further assumed that, quite generally, the objects of our perception are the causes of our perceptions. If we add the premise that digital objects cause the perceptions of virtual objects, the identification follows (2017: 318-9).

Finally, the terraform argument (2017: 343-4) supports the evaluative component of virtual realism. We can bracket this argument for the purposes of the following. Nor need some arguments that Chalmers lists as possible counterarguments against virtual realism detain us. The reason is that Chalmers debunks them successfully (e.g. 2017: 320).

The explicit arguments in favor of virtual realism thus support parts of realism, but assume other parts of it. The dialectical situation may thus be charitably reconstructed as follows: It is natural to interpret virtual reality in terms of virtual objects, their properties and events involving them (e.g. 2017: 329-30, 335). The argument from causal powers then shows that we have to identify the virtual objects with digital objects, as virtual digitalism has it (VR-M1). It is also natural to say that we perceive virtual objects. If we assume so, the argument from perception shows once more that virtual objects are digital objects. Based upon this view of virtual objects, Chalmers constructs properties and events involving the virtual objects. They yield the truth of the second metaphysical claim (VR-M2). Restricting the attention to experienced users of virtual reality, the epistemic commitment becomes true as well (VR-E).

Realism with respect to a certain domain of discourse has typical opponents. Chalmers takes it that his main opponent is a fictionalist (2017: 315), who holds that virtual cats are part of a fiction. ${ }^{3}$

\footnotetext{
${ }^{3}$ It's actually not clear why a fictionalist has to reject VR-V once it has been purged from its realist presuppositions. It's not incompatible with fictionalism
} 
So far for Chalmers's story about the cat. But will it really convince Tina that she got a real cat-like object?

\section{From virtual reality to computer simulations}

\subsection{Establishing a parallel}

Tina's father is a computer scientist. He helps natural and social scientists to develop and run scientific computer simulations. ${ }^{4}$ In the simulations, e.g. molecules, cosmic structures or markets are simulated. This is to say that computer programs are run that deliver proxies to solutions to mathematical equations. The equations have an empirical interpretation in terms of molecules, cosmic structures, or markets. In fact, the equations constitute the core of models of their respective target systems. The models are often much idealized, and the computer typically doesn't really solve the equations exactly; nevertheless, simulations of this kind are often used to predict and to understand the dynamics of the respective target systems.

When simulation scientists describe their simulations, they typically tell a story that describes what's going on in the model. To expand just one example, regarding simulations of cosmic structure formation, they would say that the dark matter assumed to fill the Universe is traced using so-called dark matter particles (see Bertschinger 1998 for details). The dark matter particles have a specific mass and are almost homogeneously distributed in space at an early stage of the Universe. They then interact via gravitational forces. What cosmologists observe in the simulations is that tiny deviations from the otherwise homogeneous distribution of the particles grow to form large matter clumps that are interpreted as galaxies or galaxy clusters.

Solutions to this model are evaluated using a simulation program, which outputs lists of numbers specifying the positions of the dark matter particles at the times considered. The output "data" are often

to claim that engaging with e.g. virtual cats in VR environments is as valuable as engaging with real cats.

${ }^{4}$ For the sake of brevity, I will sometimes only speak of simulations, when referring to computer simulations. 
visualized using plots that show how the matter distribution in the Universe looks like from a certain perspective. One can also construct animations that show the evolution of the structures or that allow one to "travel" through the Universe. ${ }^{5}$

When Tina's parents try to explain to her that, according to virtual realism, she has not just been given a headset, but also a virtual cat, Tina turns to her father: "If I now own a real object with my virtual cat, then you deal with virtual galaxies and markets as real objects on a daily basis."

Tina has a point. To stick with our example, suppose that, in a simulation of cosmic structure formation, a clump of dark matter particles arises that has the mass and shape of a certain type of galaxy. The working scientist observes it on the screen and takes it to be a simulated galaxy, maybe even a counterpart of a real galaxy. ${ }^{6}$ Tina's point then is that, if Chalmers's virtual realism is on the right track, the simulated galaxy too qualifies as a virtual object that is really there etc. Here is why virtual realism applies to the simulated galaxy: We may regard the virtual galaxy as a data structure and say that it is somehow grounded in physical processes on the computer hardware, so it is a digital object (VR-M1). ${ }^{7}$ The virtual galaxy has certain virtual properties (e.g. a certain mass) and is involved in virtual events (e.g. mergers of galaxies; VR-M2). Moreover, if the working scientist looks at appropriate pictures or movies of the galaxy and is aware that the galaxy is part of a simulation, she can be

${ }^{5}$ Check e.g. https://www.youtube.com/watch?v=YjUICiYlCYE (last checked June 11, 2019).

${ }^{6}$ Here, "simulated galaxy" means a computer-generated galaxy part of a computer simulation, and not a real galaxy that has been considered in a computer simulation. Note that some computer simulations have specific real-world objects, e.g. specific galaxies, as targets, while other simulations do not refer to specific real-world systems (but rather simulate a couple of possible galaxies; cf. 2017: 334).

${ }^{7}$ Strictly speaking, in a computer simulation of cosmic structure formation, a galaxy does typically not correspond to a data structure such as an array since the information about the galaxy is not stored in one array of data. The reason is that the galaxy in the simulation does not consist of a fixed set of dark matter particles; rather, particles can escape the galaxy or become absorbed by it. Chalmers (2017: 317 ) is aware of this problem, and I'll not use it to argue against him, but rather focus on examples in which virtual objects do correspond to data structures. 
said to have non-illusory perceptions of the virtual galaxy (VR-E). It thus seems that, if virtual realism works, it extends to the simulated galaxy and to all kinds of simulated entities alike.

Does it really? There are some differences between virtual reality environments and scientific computer simulations. Let us discuss whether they have an impact on the applicability of virtual realism, provided that the latter holds true for virtual reality environments.

A first striking difference is that most scientific computer simulations are not used to produce perception-like states in headsets. In fact, most often the results of computer simulations are not fed into headsets at all; if the results are visualized, the pictures are often poor and not animated. They are typically observed on a screen such that scientists will be aware of the screen. It may thus be argued that the settings simulated in scientific computer simulations are not immersive in the sense of Chalmers and that virtual realism only applies to virtual environments, which are immersive. In more detail, it may be said that, due to the lack of immersion, the simulated galaxies are not really observed, which is to reject VR-E. It may further be argued that they don't instantiate properties such as being virtually bluish, if nobody observes them, which is to reject VR-M2. It may finally be claimed that, due to the lack of a rich visualization, we cannot call the simulated galaxies real objects, which is to deny VR-M1.

But all this is not convincing. The difference in terms of visual presentation doesn't make a real difference to virtual realism. For one thing, when astronomers observe real galaxies, they are typically sitting in front of a screen and looking at pictures that look like the ones of simulated galaxies. If they can be said to see the real galaxies, which is not implausible (see Shapere 1982 for a discussion), then why not say that the simulation scientists see simulated galaxies? For another thing, whenever simulated objects are not at all visualized or only very poorly so, it is still true that the simulated objects may be visualized or better visualized and used to produce images in a headset. This option is available because there are standard means to produce visualizations and to feed them into suitable headsets. If the crucial outputs of the simulation program are stored e.g. in a file, then the simulated galaxies may be visualized without any need to change the simulation program. This possibility suffices to grant the possibility of perception and the truth of VR-E. (I here read VR-E is 
a hypothetical conditional: If somebody has experiences of a virtual object, the corresponding mental states are non-illusory.) This is also sufficient to ascribe simulated objects all kinds of properties (VRM2) because, if we follow Chalmers's argument, the instantiation of properties like colors only depends on the conditional that certain experiences are produced, if a suitable headset is used (2017: 322). It may be objected that virtual realism, in particular VR-M1, nevertheless only applies to objects that are in fact perceived via headsets. But this doesn't seem what Chalmers thinks (2017: 349). Also, this would lead to an extremely weird version of realism according to which the objects claimed to be real only exist if they are perceived. This is in fact a sort of idealism. The conclusion then is that lack of factual visualization and poor quality of visualization don't make a difference for virtual realism.

A second striking difference is that computer simulations are typically not interactive. True, the simulation scientist is free to set the initial conditions of the simulation. This activity of setting up the simulated world is sometimes compared to intervention typical of experiments (see Beisbart 2018: 177, 194-7 for a discussion). But in most simulations, computer scientists do not interfere with the simulated system, once the simulation has been initialized. In fact, most simulation programs don't allow for such interference. It may thus be said that virtual realism, in particular VR-M1-2, cannot be established for simulated galaxies and the like since no interaction is possible.

But again this is not convincing, and lack of interaction doesn't make a difference to virtual realism. One possible strategy for arguing this point would be to say that a simulation program may easily be changed as to allow for interaction. This may be thought to show that interaction is possible, and more cannot be required because, even in a virtual environment of the sort Chalmers considers, the user may as a matter of fact decide not to interact with the virtual environment, which is to say that factual interaction is not the point. But, maybe, this strategy stretches things too far because, for most computer simulations, it is natural to say that the user lacks the ability and thus the possibility to interact. A related worry is that we wish to establish virtual realism for real simulations and not for suitably modified ones.

A more promising strategy is to recall the way in which Chalmers makes a case for virtual realism. There, interaction is crucial for 
defusing worries to the effect that engaging with a virtual world is as idle as Nozick takes it to be to be hooked up to the experience machine. Otherwise, interaction doesn't much matter for Chalmers's argumentation. In particular, the construal of virtual objects and their having properties etc. is not affected. Nor are the arguments from causal power and perception. So why not say that computer simulations contain virtual objects too?

In his lectures, Chalmers considers possible extensions of virtual realism e.g. to mixed environments (2017: 344-8). The extensions that he accepts allow for interaction - this is certainly so with mixed environments and interactive novels, and arguably also for lucid dreams and hallucinations. But no systematic reason is given why virtual realism should be restricted to environments that allow for interaction. ${ }^{8}$ Chalmers may only resist the extension by saying that virtual realism is more natural for virtual environments if interaction is possible. But such an appeal to naturalness wouldn't carry conviction. First, it is very natural to describe computer simulations in terms of the underlying model and its constituents, e.g. galaxies. Second, what appears natural is often a matter of context as well as education and training and cannot make a decisive difference to matters of metaphysics. ${ }^{9}$

${ }^{8}$ Indeed, Chalmers allows for "improper" virtual reality environments for which one or the other defining characteristics of proper virtual reality is missing (2017: 313-4). It is not entirely clear to what extent virtual realism is supposed to apply to improper virtual reality environments. If it extends to improper virtual reality, then clearly interactivity cannot make a decisive difference. An analogous point applies to other differences on my list that only turn on one the three defining characteristics of virtual reality.

${ }^{9}$ As far as interaction is concerned, there is an interesting parallel with the debate about scientific realism, i.e., the debate whether we have knowledge about unobservable entities and their properties. Hacking (1983) has famously argued that what really made a positive difference for knowledge about, say, electrons was our ability to interact with them. He thinks that we can claim knowledge about unobservable entities, if we "manipulate them using well-understood lowlevel causal properties" (1983: 274). Likewise, virtual realists may claim that we are entitled to take virtual objects as real if we interact with them. But Hacking's criterion doesn't do much work in the debate about scientific realism. To say that we manipulate electrons is to describe some of our actions in terms of a certain vocabulary, which apparently refers to unobservables. Skeptics with anti-realist 
A third noteworthy difference between virtual reality environments and computer simulations is that the latter often cover unobservable aspects of reality. This is to say that the equations to which the simulations yield approximate solutions often refer to characteristics the instantiation of which cannot be observed (unobservable characteristics, for short). Very often, these characteristics will be those of unobservable objects, e.g. the distances between, or the socalled color charges of, quarks (see e.g. Bissey et al. 2007 for simulations of quarks). It may now be said that virtual realism does not apply to simulated unobservable objects and their unobservable characteristics. For one thing, how can we talk about non-illusory perception of virtual quarks (VR-E), if real quarks cannot be perceived, either? For another thing, it may be claimed that the metaphysical components (VR-M1-2) of virtual realism become implausible when we talk about unobservables and their unobservable characteristics.

But once more, the argument doesn't carry conviction. Note first that, if Chalmers's construction of virtual objects and properties works (cf. VR-M-1), it does so for unobservable objects as it does for observable ones: We may regard the objects as data structures and say that they are constituted by certain processes in the computer that realize computations referring to the objects. There is indeed no difference in the way in which observable and unobservable entities are dealt with in computer simulations.

Note also that it would be narrow-minded to restrict virtual realism to objects that have visible counterparts. After all, many people (at least scientific realists) don't restrict things to observables, so why do this with virtual objects?

Nor is there anything that prevents us from applying Chalmers's understanding of properties (cf. VR-M-2) to unobservable characteristics. That unobservable entities have some such characteristics

leanings may simply ask: Why is it legitimate to use this vocabulary and to take it at face value, viz. as referring to unobservables? Hacking can only convince anti-realists using his criterion if he can mount an independent case for using this vocabulary and for taking it literally. But this brings us back to other arguments in favor of scientific realism, and thus, the possibility of interaction does not suffice as an independent argument for scientific realism. If my argument above is correct, the possibility of interaction doesn't make a decisive difference for virtual realism, either. 
can even be made visible using standard visualization techniques. ${ }^{10}$ For instance, that a certain agglomeration of quarks has a particular shape can be visualized by showing the shape like the one of a visible object. The simulated agglomeration thus has the power to produce shape-like appearances in the headset, which is to say that it has a certain virtual shape, at least if we assume Chalmers's understanding of virtual shapes. Recall also that Chalmers allows at least some virtual properties to be identified in terms of their roles they play in the virtual world. Color charges and similar unobservable characteristics of quarks do play specific roles in respective computer simulations.

As far as perception and VR-E are concerned, we can argue as follows: In some simulations with unobservable objects, these objects are in fact visualized. So we can say that the virtual objects can be perceived. It is of course true that the perception of virtual objects is somehow special. For instance, spatial patterns with characteristic lengths significantly smaller than the wavelength of visible light do not have parts with different colors. Nevertheless, in a visualization, the parts of the pattern are most naturally marked by different colors. So the visualization is in some sense deceptive. But experienced users of the visualizations will know about this problem. They will thus not be deceived, but rather understand that the colors are just representational means to communicate the spatial pattern. In cases of other simulations in which unobservable entities and their unobservable properties are not visualized, we may say that non-illusory perception of them qua virtual objects is at least possible because there are standard means to visualize them. Also, as I have argued above, it is not part of Chalmers's virtual realism that virtual objects must be perceived.

This shows not only that all kinds of computer simulations feature virtual objects. It also makes clear that many virtual environments contain more objects than one may think. If Tina's virtual environment is based upon a computer program in which e.g. the air

${ }^{10}$ Check e.g. http://www.physics.adelaide.edu.au/theory/staff/leinweber/ VisualQCD/Nobel/index.html (last checked June 11, 2019) for visualizations of simulations based upon quantum chromodynamics, which is the theory about strong interactions between quarks. 
molecules are traced, then the virtual environment contains virtual molecules. It would be at least as unnatural to exclude them from being virtual objects as it is to deny the existence of real unobservable objects such as real molecules. In fact, certain skeptical worries about underdetermination that one may have in view of our knowledge of unobservable entities are inappropriate here. For, if a computer simulation traces the behavior of certain unobservable entities, this is encoded in the simulation program. There are thus matters of fact about what virtual air consists of, and they can be known by analyzing the computer program. It is thus not sensible to worry about the possibility that virtual air may be made out of entirely different stuff and that the unobservable virtual reality is beyond our grasp.

A fourth and final difference between at least some scientific simulations and virtual environments is that the former are based upon highly idealized models. For instance, in some multi-agent simulations, agents are represented only using a utility function for a particular choice and a spatial location (think of Schelling's famous model for segregation and related implementations on the computer; see e.g. Schelling 1969). This is certainly not enough information to produce an image showing an agent. One may thus think that e.g. agents in multi-agent simulations are too poor to be virtual objects.

But once more, this would put an unnatural restriction on virtual objects. In virtual environments, many objects are only traced using few characteristics too. Further, the number of characteristics of an unobservable object that are traced in a computer program can often be chosen from a broad range of options. It seems arbitrary to draw a line and to say that some objects traced by computer programs form virtual objects, while others do not because they have too few characteristics. As a consequence, we should accept „poor“ virtual objects.

It is true that the idealized agents in multi-agent simulations are not virtual human beings because, in virtual reality, virtual humans have to fulfill exactly the roles that humans fulfill in reality, and idealized agents do not do so (cf. Chalmers's draft, Sec 4, p. 14). But this does not imply that the agents are not virtual objects.

All this shows that the differences between scientific computer simulations and virtual environments are insignificant for the justification of virtual realism. Thus, if virtual realism is on the right track for virtual reality environments and if Chalmers's construal 
of virtual objects and their properties makes sense, it carries over to simulated objects from scientific computer simulations. Chalmers does in fact say that computer simulations are behind virtual reality (2017: 313), and it is natural to apply virtual realism to all sorts of computer simulations and the objects figuring in them.

Now at first sight, all this may not seem bad news for virtual realism. For doesn't it mean that the position has broad scope? But at closer analysis, the prospects of virtual realism become bleaker, when we consider the extensions I have conditionally proposed. The reason is that, intuitively speaking, it seems implausible to think that all sorts of simulated objects really exist. For one thing, there are so many simulations with so many simulated entities, and do we want to admit so many real things in our ontology? For another thing, what figures in a simulation is often quite remote from reality and highly idealized. We have particles devoid of any extension, massless levers and what have you. Should we really say that these things all exist?

\subsection{Dealing with an objection}

Chalmers may object that there is nothing problematic if e.g. simulated galaxies too are virtual objects, and thus, according to virtual realism, real entities. To this purpose, he may argue that I appeal to intuitions that are ultimately misguided. He may also try to defend his position by pointing out that virtual realism adds only commitments to virtual objects.

But such an objection wouldn't work. To begin with the last point, note that, for Chalmers, virtual objects are indeed real objects, because virtual means "computer-generated" and not just "as if, but not really existing" (cf. 2017: 311). Some opponents of virtual realism deny that virtual cats are real objects. So virtual realism does lead to a proliferation of objects in comparison to other positions.

Note further that my argument is not only based upon intuitions. Rather, I appeal to an important theoretical virtue: Ceteris paribus, a theory is preferred to another one if it postulates fewer entities than the latter. Now, as we have just seen, virtual realism, as defined by Chalmers, does claim that there are entities the existence of which is denied by other parties. This proliferation of things is a pro tanto reason to reject virtual realism. This reason may be outweighed by 
other reasons; e.g. if virtual realism can explain things that other positions fail to explain, or if it explains things much better than do other positions. But it is doubtful whether virtual realism does so. What virtual objects can possibly explain are (i) virtual events and (ii) the experiences of objects and of events on the part of users. But it is controversial between virtual realists and their opponents whether there are any virtual events, so we have to bracket their explanation. The experiences of objects and of events on the part of the users may be explained in alternative ways too, for instance by pointing to processes in the hardware and the headset. It may be objected that this explanation is less appealing and more complicated than an explanation in terms of virtual objects. Even if this is correct, it is far from clear that related benefits in an explanation in terms of virtual realism outweigh the costs of proliferating things.

As far as intuition is concerned, we can back up intuitions that resist the proliferation of objects, when we compare computer simulations to other applications of computers. Computer programs too are used to evaluate integrals, to determine roots of functions etc. Now from a computational perspective, the computations underlying such applications and underlying computer simulations are not very different. Likewise, from a physical perspective, there isn't much of a difference. When we consider the physical processes in a computer hardware, it is extremely difficult to tell whether a simulation is run or whether a different computation is carried out. Even as far as visualization is concerned, there need not be much of a difference; some algorithms that aim at identifying a root of a function can be used to produce animations too. This faces virtual realists with a dilemma: They either allow for even further virtual objects (e.g. virtual roots), which seems even more implausible (what kinds of objects are virtual roots?). Alternatively, they draw a line between computer simulations and other uses of the computer and only allow that the former give rise to virtual objects. But this seems like drawing an artificial distinction.

The latter point may be seen by considering an interesting borderline case, viz. a computer-based calculation that may be interpreted as a simulation, but is in fact not regarded to be one. For instance, a Monte Carlo integration that is supposed to return the value of some area may be interpreted in terms of a real process in which a person 
throws little objects, say pebbles, on the area and its surroundings, but is usually not interpreted in this way (cf. Buffon's needle experiment; see Grüne-Yanoff and Weirich 2010: 29-30 for a discussion). Are there then virtual objects thrown on an area according to virtual realism? However we answer this question, a problem for virtual realism arises. If the answer is yes, what prevents us from obtaining more and more virtual objects simply because we can tell stories around all kinds of computations done by a computer? If the answer is no, then it depends on the way a computer program is understood by the user (e.g. the working scientist) whether there are virtual objects or not. But it seems weird that the existence of virtual objects turns on the view of the user.

Chalmers may try a slightly different route to object against my point that virtual realism leads to an undue proliferation of things. He may want to argue that even a rival, fictionalist position does not avoid the proliferation of things. After all, isn't it the case that fictionalists have to admit the existence of e.g. virtual cats in some sense too?

To show that this is wrong, I'll consider a specific form of ficitionalism about virtual objects (such a position is proposed by McDonnell and Wildman, forthcoming in this volume). The idea is that virtual objects are parts of a Waltonian game of make-believe (Walton 1990). To understand this, consider first Walton's famous example of a game of make-believe (which does not yet involve computers; 1990: 21-8): Children agree that stumps in a wood count as bears in a game. Depending on what is true about the stumps, we obtain fictional truths about bears, e.g. that there are about 30 bears in the wood etc. For Walton, the stumps are so-called props (1990: 37-8). They help to grant truths in the fiction. What we need for this account, ontologically speaking, are props, but not bears and no bear-like objects apart from the props.

If we wish to apply this view to objects from virtual reality environments and computer simulations, we can say that they form part of a game of make-believe that arises because people are prepared to treat certain props as virtual cats, simulated galaxies etc. A natural suggestion in this respect is that the computer-generated images of virtual cats and simulated galaxies act as props. Alternatively, we 
may consider certain processes in the hardware as props. ${ }^{11}$ In either case, the props are real-world objects that exist in the world anyway. Virtual realists and their fictionalist opponents agree that there are e.g. cat-like images on the screen. Virtual realists claim that there are additional objects, viz. virtual cats that explain the images on the screen. Fictionalists, by contrast, deny that there are any other real objects apart from the props. So virtual realists are committed to the existence of objects that Waltonian fictionalists deny. Of course, fictionalists must say that some objects are not just objects, but also props. But this does not enrich the ontology. It's simply to say that certain things are treated in a particular way. So at least certain types of fictionalism can do with fewer objects than virtual realism.

All in all, the objection that the proliferation of objects implied by virtual realism isn't problematic does not carry conviction. Virtual realism does postulate more entities than do other positions, and this is a problem, among other things because parsimony is a theoretical virtue.

\subsection{Using the parallel with computer simulation}

Granted that virtual environment technologies and computer simulations are very similar, we can use the parallel to import insights from the philosophy of computer simulation to the philosophical analysis of virtual reality environments. In what follows, I'll sketch a view of what the status of simulated galaxies etc. is. This suggests an alternative to Chalmers's virtual realism. It also puts some pressure on the latter because a natural position about the status of simulated objects is quite different from Chalmers's position.

It is a commonplace in the literature about computer simulations (see e.g. Humphreys 2004: ch. 5 and Winsberg 2010 for pioneering accounts) that they are closely associated with models. In fact, "to simulate" means roughly "to model". Also, many computer simulations are based upon prior models, e.g. the so-called Ising model. Further, each simulation can be said to implement a model.

Modeling is a research strategy with distinctive characteristics

${ }^{11}$ It is less problematic to treat a process as an object in a game of makebelieve than to claim that a process is an object. 
(e.g. Suárez 2004, Weisberg 2007). The strategy is to not investigate a target system directly, but rather to first consider a substitute, which is often called source or simply model, to analyze the latter and to translate the findings from the model to the target. This is certainly true of two broad classes of paradigmatic models, viz. material models, e.g. scale models of cars, and fictional models, i.e., imagined systems such as a system with point particles that interact with each other via Newtonian gravity. Note that these types of models are distinguished in terms of the ontological status of their sources. Note also that the term "fictional model" needs to be understood with some care. The idea here is not that fictional models function like fictions in every respect. The point is rather that they are merely imagined systems which "would be concrete if they were real" (see e.g. Godfrey-Smith 2006: 734-5 for this formulation) and which are used to study a target.

Sometimes, other sorts of things, e.g. viewgraphs or mathematical equations with an empirical interpretation, are called models too. But viewgraphs and equations immediately depict or describe (real or imagined) systems. If they depict or describe the target system (at least to some approximation), then they do not really instantiate the indirect research strategy characteristic of other types of modeling. If they describe a system distinct from the target system, the latter is used as a surrogate of the target system, so they depict or describe a model of the target. We should then call the viewgraphs and the equations "model descriptions" and differentiate them from the models themselves (see Weisberg 2007: 217 for this distinction). ${ }^{12}$

When computer scientists describe their simulations, they are typically talking about their model. They talk e.g. about dark matter particles, about the galaxies they form and so on. The crucial question now is what ontological status these objects have. Since models are classified according to the ontological status of the source, the question is: What type of model is associated with a simulation (see Beisbart 2014 for the following)? Clearly, the objects do not form

12 The distinction between models and model descriptions is not uncontroversial; nor is the claim that equations are not models, strictly speaking. But in what follows, the argument does not hinge on the assumption that equations are not really models. What I assume though is that animations from computer simulations are model descriptions. This seems plausible. 
a material model because nothing physical like a galaxy is involved in the simulation. Rather, if the equations that the simulations try to solve to some approximation refer to a system distinct from the target (e.g. because the equations are based upon so many idealizations that they cannot refer to the target directly), they describe a fictional model of the target. This suggests that the simulated cosmic structures etc. are part of a fictional model, i.e., a merely imagined system, and that we are not talking about real objects. This is very natural because a lot of simulations are in fact built upon prior models that certainly are fictional or imagined systems, e.g. spins distributed in a two-dimensional grid in an Ising-type model.

It may be objected that there are different cases of simulations in which the equations solved by the simulations directly yield a description of a real target. But if there are such cases, there is no need to refer to any object beyond the target, and we can bracket such simulations in what follows. The point then stands that all kinds of simulations are well analyzed without postulating a layer of separate, but non-fictional simulated objects. ${ }^{13}$

But there is a complication for the simulations that primarily refer to an imagined system. It is plausible to say that each simulation of this type is not just associated with one model, but rather with several ones (cf. Winsberg 2001). The reason is that scientists often start with a prior model independent from the simulation. To obtain approximate solutions using the computer simulations, the scientists have to modify the model. For instance, if the dynamics of the source is described in terms of differential equations, the equations need to be discretized. This is naturally captured by saying that the model is changed. If the simulation program is run on a hardware, additional deviations from the original model can arise, for instance, round-off errors are incurred during floating point operations. We may conceptualize this by saying that, what has really been solved, is a yet different model, call it the computational model (cf. Schlesinger et al. 1979). So we obtain a series of models. Clearly, all of them have

\footnotetext{
${ }^{13}$ Note that there are intermediate cases between the types of simulations just distinguished: A computer simulation may refer to a real system but consider it under very counterfactual circumstances or mix it with unrealistic stuff (2017: 344-6). For the purposes of this paper, we can handle such cases as we do with the first class of simulations.
} 
the same status, and they must all be fictional models if some of them are such, as suggested above.

A virtual realist à la Chalmers will have to pick one of the models and to say that its objects are real, that the latter really instantiate properties etc. She will most likely choose the computational model because this is the model that really produces the outputs.

From the perspective of the discussion about computer simulations, then, what Chalmers essentially does is to say that objects in some (viz. computational) fictional models are real because the models are implemented on a computer and because they produce output accessible to the senses. This is quite unnatural given that there are often other models associated with a computer simulation. Further, what's the point of claiming that the objects in some simulation really exist qua digital objects?

To summarize this section, the upshot is first that, if virtual realism holds true, it should be applied to computer simulations, too. Intuitively, this goes some way towards a reductio: We do not want to allow for so many objects, especially "poor" ones, as familiar from fictional models. The literature about computer simulations indeed suggests an alternative picture: If computer simulations do not directly refer to the real world, they refer to fictional systems. Simulated cosmic structures etc. are fictional systems used to understand a target. What the computer simulation does is to deliver handy model descriptions. Philosophers of science have to allow for such model descriptions anyway if they want to make sense of the practice of modeling. So why say that the imagined objects are real simply because there are handy descriptions and nice pictures of them?

All this does not show that virtual realism is false. But it casts some doubts on it and suggests that something might have gone wrong with the way in which Chalmers has constructed the position. In what follows, I will elaborate this diagnosis by analyzing the steps with which Chalmers builds up virtual realism. I'll take the metaphysical and epistemic components of the position one after the other, and analyze the support they have. The main gist of my criticism is that Chalmers allows himself to construct a realist position too easily by a number of problematic moves. 


\section{Realism easy-going}

\subsection{Virtual objects (VR-M1)}

Chalmers starts with objects. The first claim within his realism holds that virtual objects really exist, and his defense of virtual realism starts from objects too (2017: 317). But what does it mean to say that virtual objects such as avatars really exist? In philosophical circles, there has been increasing concern about claims about unrestricted existence. Quine (1948) identifies objects relative to theories only. More recently, Putnam (2002) has argued that ontology is ill-conceived if it is supposed to find out what objects there really are. There is no unique way to identify objects, and questions of the sort "How many objects are there in this-and-this realm?" can only meaningfully be answered, if he have agreed upon a restricted sense of there being objects - e.g. qua fundamental material particles. Consider now a paradigmatic fictional entity such as Sherlock Holmes. Does he exist? Well, in some way he does, in others not. Presumably, the same is true about objects in virtual reality or simulations. Just to insist that virtual objects exist doesn't mean much.

Now Chalmers has a lot more to say about virtual objects. In particular, he claims them to be digital objects. To some approximation, which will suffice for our purposes, the latter "can be regarded as data structures" (2017: 317). Further, for Chalmers, digital objects are "constituted by computational processes on a computer" and "are grounded in computational processes" (ibid.). But does this answer the question of what digital objects really are?

Turn first to the claim that digital, and thus virtual, objects are, roughly speaking, data structures. In the literature about programming, "data structure" is a technical term roughly referring to ways of organizing data; for instance, queues and stacks are well-known data structures (see e.g. Brass 2008: xi). Data structures in this sense arise most naturally at the level of a computer program. But programs are ultimately sets of instructions and thus abstract objects. They do not have causal powers and thus cannot form objects with causal powers. So Chalmers is presumably more interested in something like the following. In a computer simulation (be it one that 
underlies virtual reality technology or a scientific simulation), objects are traced using characteristics, e.g. position, mass, color etc. When the simulation program is executed, the characteristics take values that change with time. The computer hardware is used to store and further process the values. This means that, for a specific state of the hardware at some time, there is something about the hardware that corresponds to the fact that the characteristics of the object (position, mass, color) take such and such values. This is the basis on which digital objects must be constructed.

The problem now is that, at the level of the hardware, it is difficult to identify anything object-like that may properly be regarded as digital (and thus virtual) object. The computer is built up of processors, which sequentially store and process information about various characteristics traced by the computer program. On all-purpose machines, there are typically relatively few processors, and none of them is exclusively reserved for the numbers that trace one particular object in the simulation. The only way to link virtual objects with the computer hardware is to say that certain aspects of the computer state, or, maybe, of the dynamics of the computer hardware correspond to the object. But does this tell us what the virtual objects are? Can we identify the virtual objects with those aspects? To say that objects are aspects of states or processes sounds like a category mistake.

The background to this problem is as follows. A computer simulation can be described at various levels (Barberousse et al. 2009). To characterize a simulation, we can use a fictional model, the mathematical equations to be solved, the algorithm, the program or the physical processes during which the program is executed. We can easily identify objects like cats at the top level (i.e., the fictional model). But to obtain physical objects with causal powers that can be digital objects and produce e.g. appearances of colors, Chalmers has to move to the lowest layer of physical reality. Now the relationship between this layer and the top layer is very complicated. The reason is that the bottom layer realizes a computation that solves equations that in turn describe the virtual cat or whatever it is. As Barberousse et al. (2009: 563) point out, the relationship is semantic. Certain physical processes are interpreted as doing calculations due to conventional mappings between aspects of hardware states and the fictional model. For this reason, the relationship cannot be easily 
described in purely physical terms. Since there is no mapping that is cast in simple physical terms and that connects the fiction and the computer hardware, we cannot identify counterparts of the fictional objects in plain physics terms. But this means that there are no such object-like counterparts in the physical world, for we would have to carve them out using simple physics terminology, which is tuned to what we take to be physical objects.

It may be objected that virtual realists don't need physical $o b$ jects to make sense of virtual objects qua digital objects. But why then insist that there are virtual objects which are supposed to be digital objects with causal powers, if we can just say that there is an extremely complicated relationship between the fictional objects and some physical stuff?

Now apart from roughly identifying virtual objects with data structures, Chalmers also proposes to say that virtual objects are grounded in, or constituted by, computational processes. This is significantly weaker than any identification of virtual objects with computational processes or whatever. And it seems less of a problem to say that objects are grounded, or constituted by, computational processes (although the idea that processes constitute an object sounds still a bit strange).

The problem though is that claims about grounding or the constitution of objects do not answer the question of what the objects are. To know what certain objects are we should at least be given the category they belong to as well as some of their essential features or at least the types of features they must essentially have. For instance, when the first atomists claimed that the material world was made out of little invisible particles, they could say that these particles were material bodies, and they could provide determinable features the particles would have (e.g. mass). ${ }^{14}$ They could also point out analogies to the observable world (e.g. to little balls). Chalmers's virtual realism, by contrast, strikes me as poor on these counts. He doesn't really specify the category of virtual objects. He does provide features that virtual objects can have, but they are all virtual properties. Appeal to such properties is not very promising because the question

\footnotetext{
${ }^{14}$ Today, there is of course a real issue of what particles like electrons etc. really are because they are quantum-mechanical objects and because quantum mechanics is so difficult to interpret. This is why my example is about early atomism.
} 
of whether there are such properties is part of the debate on virtual realism. It is true that properties like virtual color are explained in terms of analogies. But the problem is that all virtual properties to which Chalmers appeals are ultimately defined in terms of their effects. This means that describing these properties doesn't really get us closer to the virtual objects themselves. ${ }^{15}$

To strengthen my case, let me recall what the dialectical situation is like. Chalmers claims that there is a layer of virtual objects, while other authors deny that layer. Now to say that the supposed objects are grounded in, or constituted by, something else doesn't really add to the plausibility of the position. ${ }^{16}$

All in all, I conclude that Chalmers's virtual realism is too cheap because he doesn't have a satisfying answer to the question of what digital, and thus virtual, objects are. An identification of virtual objects with data structures doesn't work, and what he has to say about the constitution or grounding of virtual objects falls short of answering the question of what the objects really are. ${ }^{17}$

\footnotetext{
${ }^{15}$ It may be claimed that there is in fact a good analogy as follows: Virtual objects arise from the hardware of a computer in the same way as mental states arise from the brain. But the relationship between the brain and mental states is not well understood. Also, at the level of the mental, many authors do not assume there to be mental objects (rather, they prefer mental states or processes), while virtual realism needs objects. Note further that there is a strong epistemic disanalogy between both cases: Whereas we know that there are mental states, the existence of virtual objects is disputed. We have thus no worries to admit that there are mental states, simply because we know about them. By contrast, virtual realism would have to show that there are virtual objects.

${ }^{16}$ Kripke (1980: 110-3) argues that, for some objects (e.g. the Queen, this wooden table), their origin is essential to them. If this is true about artifacts, the origin of an individual artifact is at least part of its identity. Likewise, the origin of a virtual object may be part of its identity. If this is true, then specifying the way in which a virtual object is grounded goes some way to explaining what this object is. The question though is whether it goes far enough. The specification of some essential features of an object doesn't necessarily tell us what this object (or its essence) is simply because there may be additional essential properties. Note also that Kripke is concerned with individual objects. Our concern, by contrast, is a whole class of objects (virtual objects). If it is not clear what these objects are, it does not much help to assert that they essentially have a certain type of origin or grounding.

${ }^{17}$ In this special issue, McDonnell and Wildman (forthcoming) face Chalmers
} 


\subsection{Virtual properties and their instantiation (VR-M2)}

Turn now to virtual properties and their (virtual?) instantiation. In the Petrus Hispanus Lectures, we find two slightly different strands in the characterization of virtual properties. First, virtual colors and virtual spatial properties are, roughly speaking, claimed to be dispositions to produce impressions of colors and spatial properties under the conditions typical of virtual reality (2017: 321-3). Virtual properties of this type would have to qualify as secondary qualities because their specification refers to the way we know of them. As such, they arguably violate the independence claim constitutive of many brands of realism requiring that the domain of things under consideration be mind-independent or objective (see e.g. Wright 1993: 1-2 and Psillos 1999: 12-3 for the independence dimension of realism; whether, and to what extent, secondary qualities really violate the independence part of realism is admittedly a tricky question).

Second, Chalmers characterizes at least some virtual properties more generally in terms of the roles they take in virtual reality. For instance, virtual shapes are said to fulfill the same role that real shapes have in the real world (2017: 323-4). This idea may be generalized to virtual properties with no counterparts in the non-virtual world by saying that they have distinctive roles to play in virtual reality (cf. 2017: 349). Note though that this characterization does not coincide with the first one, if we focus on colors. According to the first characterization, reactions of users are decisive for virtual colors. But if virtual colors play the same functional role in virtual reality as colors do in reality, then they would have to produce impressions in virtual humans, which is something else (and which they don't do unless virtual humans have consciousness), and do a couple of other things (viz. produce those effects in the virtual world that real colors have in the real world).

A more significant worry is that Chalmers obtains virtual properties and their instantiation too cheap. To say that virtual properties are dispositions to produce certain impressions or that they fulfill

with a similar theoretical dilemma: Chalmers has either to identify virtual objects with digital objects or to claim a weaker dependency relation. McDonnell and Wildman argue that both options are not attractive, but give different reasons. 
certain roles isn't informative enough. To take up the first strand of thinking about properties, it is of course true that the hardware underlying a virtual reality environment produces impressions of e.g. (virtually) gray (virtual) cats, but Chalmers has to show how the instantiation of a virtual property by a virtual object produces the impression. Chalmers faces a tough challenge to explain this because it is already unclear what a virtual object really is. At some point (2017: 322), he appeals to an entry in a data structure, but this does not suffice since data structures and their entries are abstract objects that do not have causal powers.

Likewise, to take up the second strand of thinking, it is true that all sorts of roles can be distinguished at the level of e.g. virtual cats. But Chalmers is a virtual digitalist, so he has to show how these roles emerge from the level of the digital objects, and thus roughly from the physical processes running in the hardware. The problem here is once more that there is a huge gap between describing the computer simulation in terms of physical processes (and digital objects) and in terms of the simulated reality (roles in the virtual environment).

Chalmers may object that I'm requiring too much and that I expect a reductionistic characterization of virtual objects and their properties, while, as we all know, reductions are hard to obtain. However, such an objection does not seem legitimate. It is Chalmers who wants to say that virtual objects are real, and not just fictions, because they are digital objects that are somehow part of physical reality. If the computer processes in the hardware are decisive for there being objects of a certain kind (digital and thus virtual objects), it should be clear how the objects arise from the hardware and its processes, and likewise with properties. If this is not explained, it seems more natural to say that the computer delivers animations of fictional objects.

\subsection{Epistemic access (VR-E)}

Turn now to epistemic access to virtual worlds. Chalmers thinks that there is non-illusory perception of virtual objects. Now if virtual objects are digital objects, then users of VR techniques would have to observe digital objects. But can we say that digital objects are observed? Let's grant for the sake of argument that there are digital 
objects and that they are some proximate causes of the appearances on the part of the user. This is not enough to claim that the digital objects are perceived. For this to be the case, the appearances should also represent the digital objects, e.g. by being similar to them or in some other way. But the appearances of virtual objects do not resemble digital objects, if the latter are computer processes or something like this. And even most experienced users of VR know too little about the computer hardware as to be able to take the appearances to be representations of digital objects. ${ }^{18}$

Moreover, the claim that experienced VR users have non-illusory experiences of digital objects is established too quickly. The essential trick is to say that experienced VR users have an appropriate conception of the objects they have appearances of. They are supposed to think of them as virtual objects. But it now seems that virtual realism gets off the ground too easily. Chalmers starts from the catimpressions etc. produced by some VR technology. He tells us that cat-impressions correspond to real objects, viz. virtual cats, by appealing to parts of the computer hardware that are causally responsible for the impressions. He grants that the cat-impressions can be illusions but assures us that they are not, if the epistemic agent (the user) has the correct attitude, viz. takes the impressions to refer to virtual objects. But this recipe can easily be extended to other cases: Point to the causal history of perceptual or likewise states, postulate objects that figure in this history and claim that we do really have epistemic access to those objects, if we interpret the perceptions in an appropriate way, i.e., in terms of the objects just postulated.

This very trick can be applied to sorts of fiction, for instance. Suppose that children are playing and assuming that stumps that are in the shadow are bears (I here adapt the example from Walton 1990: 21-8). Following the recipe just given, we can construct a realism according to which some sorts of bears exist. These sorts of bears are stumps in the shadow. They are perceived in a non-illusory manner

\footnotetext{
${ }^{18}$ Note that this argument does not assume a similarity account of representation. It only presupposes that a representation is used to learn something about the object that is represented (cf. Suárez 2004). This condition is violated if digital, and thus virtual, objects are something in the hardware about which the user does not know much. Then she cannot use the images of the virtual cat etc. to learn more about digital objects (see Beisbart 2018: 181-6 for a more detailed argument).
} 
when children see the stumps and interpret them as bears. But it seems odd to construct a realism about what seems to be part of a fiction if realism is supposed to say that virtual objects are not fictions.

\subsection{Fictions}

This brings us to a more general point about fictions and fictionalism. Chalmers insists that virtual objects are not fictions (see in particular 2017: 316-7) and contrasts his realism with fictionalism (2017: 315-7). Note first that it is not the same to say that some range of things $\mathrm{R}$ are part of a fiction and to advocate fictionalism about the corresponding domain of discourse. The reason is that most varieties of fictionalism about a domain do not claim that $\mathrm{R}$ is part of a fiction. True, fictionalism is often loosely characterized as claiming that something we talk about is a sort of fiction (e.g. Eklund 2017, introduction). But this is at most a first pass at fictionalism. A more precise characterization of the common core of fictionalism is the claim that a certain discourse is not, or is not meant to be, featuring literal truths (ibid.). This does not imply that it engages in fictiontelling, literally speaking.

Consider now first the view that virtual objects are literally part of a fiction. In the philosophical literature about fiction, it is debated whether fictional characters exist or not (see Koon and Voltolini 2016). Some authors think that they do. So to say that virtual objects are fictions is not to claim that they are not real. It is thus possible to say that virtual cats etc. are parts of fictions and at the same time digital objects. True, the most common views about what fictional characters and objects are, i.e., possibilism, Meinongian views and creationism (ibid.), do not take fictions to be part of the material world (as are presumably Chalmers's digital objects). But we may then read Chalmers's position as a new way of spelling out what fictional objects can be - at least for some such objects, i.e., those generated in terms of virtual reality technologies. ${ }^{19}$

${ }^{19}$ Chalmers (2017: 334-7) concedes that some virtual reality environments are associated with a fiction. He further admits that all virtual reality environments can be associated with a fiction. So he would to some extent agree with my claim that his virtual realism is compatible with the view that virtual objects are 
Turn now to fictionalism. There are various types of fictionalism, which differ in the way in which they assign truth conditions to statements from a domain of discourse that is interpreted in a fictionalist vein. One of them is object fictionalism (Yablo 2001: 76-8). The rough idea is that a statement from the discourse has a literal, but false content F. But there is a non-literal, but possibly correct thing to say with the statement, and this is that things are such that, according to the rules constitutive of the fiction, F is true. The crucial facts to which Chalmers refers in order to justify his virtual realism can be used to establish a type of object fictionalism. The rules of the fiction have it that cat-appearances are to be treated as representations of objects. The processes in the computer then make it the case that, according to the fiction, the cat is e.g. gray.

What this shows is that the crucial facts to which Chalmers refers lend themselves to a fictionalist interpretation. But then his realism and fictionalism are not very far apart.

\subsection{Virtual worlds?}

When talking about virtual environments, Chalmers speaks of virtual worlds. Such talk sounds impressive. But at closer inspection, virtual worlds are rather poor and to some extent strange. To finish my argument, I'll briefly look at some ways in which virtual worlds are so.

First, depending on the level of detail that is covered by a computer simulation, virtual worlds can be very poor. If only the dining room is simulated, there is nothing but the dining room in this world. If the simulation doesn't resolve what's in the cupboard, then the cupboard is empty. If some period of time wasn't simulated, then there was no such time.

parts of fictions. But Chalmers thinks that in either case the digital objects have priority over the (possible) parts of the fiction because it is not necessary to interpret the virtual objects in terms of a fiction. This is sensible if we can be sure that there are virtual objects qua digital objects that are independent of any fiction. But I have above raised doubts about the existence of virtual objects. At this point, I can add the following observation: The problem about Chalmers's construal of digital, and thus virtual, objects is that some merely imagined, or fictional characters (e.g. the virtual cat) are somehow projected in the material structure of the computer hardware. This suggests that the fiction is neither optional nor secondary. 
Second, again depending on the level of detail that is simulated, virtual worlds can be indeterminate in many ways. They leave questions open that we may raise about them. There is no fact of the matter that determines the answer. For instance, what virtual stuff are the tables, chairs etc. made of? Are they wooden or made out of plastic? If a table is just modeled as a rigid body (which is a sensible option), then there is no answer to this question.

Questions that are particularly interesting in this respect concern the identity of particulars. Suppose, for instance, that the cat disappears from the dining room and a cat with the same properties comes back. Or suppose that Tina stops the simulation at some point and later starts it again. Is Tina dealing with the same cat or not? To be sure, according to Chalmers, we can assume that we are properly talking of virtual cats, because the virtual cats fulfill the roles that cats fulfill in the real world. But how are particulars identified through time using the simulations? If virtual objects are digital objects, as Chalmers thinks, then the question is how we can identify digital objects through time. Even if we assume that Chalmers can specify what digital objects are, it's not clear to me how to identify individual objects through time. Suppose, for instance, that a certain type of process in a computer hardware is decisive for the there being a virtual cat. If such a process is stopped because the simulation is interrupted and if the simulation is later resumed, are we talking about two processes? Or the same process?

Third, virtual worlds are not homogeneous, metaphysically speaking. What happens in a virtual world is partly determined by causes operating in the virtual world (virtual cats and the like), but partly also by agents outside the virtual world (e.g. Tina). Note in this respect that Tina is not a virtual object for Chalmers since she is not a digital object (unless we all live in a computer simulation). Likewise, there is an inhomogeneity regarding events: Most of them are digital events in that they only involve digital objects and their properties. But a few events that are naturally counted as part of the virtual world, e.g. Tina's decision to pet the cat are not virtual events (cf. 2017: 344). ${ }^{20}$

${ }^{20}$ I take it that this is at least part of the reason why VR-M2 is qualified with a "largely" (2017: 311). 
Fourth, to the extent to which facts in the virtual world stretch, they are not evidence-transcendent for human beings. The reason is that, for Chalmers, ultimately processes in the computer hardware determine what happens in the virtual world. This type of fact can in principle be known by humans. ${ }^{21}$

Fifth, there are some interesting problems about time in a virtual world. Take two arbitrary events in a simulation that is not interrupted (things become of course even more difficult if there is an interruption). What is the time interval between them? There is not just one way to answer this question. A first answer is provided by the time it takes the simulation to get from one event to the other. A potentially different answer arises when time is simulated e.g. in terms of clocks. Time may "run faster" in the simulation, because, in the animation, it takes only half an hour until the pointer of a virtual clock has moved from 7 to 8 o'clock. Which then is the correct period of time?

\section{Conclusion}

Where does our discussion leave us? Can we use Chalmers's virtual realism to convince Tina that she has obtained a real object not too dissimilar from a cat? Is it a sensible position?

I have first argued that, if it is sound, the virtual digitalist brand of virtual realism should be applied to all sorts of computer simulations. There would thus really be virtual galaxies etc. in scientific computer simulations. This produces a slippery slope for virtual realism because the latter doesn't look so plausible any more in view of such examples. The philosophical literature about computer simulations too suggests an alternative picture: When we describe the simulation in terms of cats, galaxies and the like, we refer to an imagined system and in this sense a fiction. The computer simulation furnishes handy descriptions of this fiction.

I have then analyzed the way in which Chalmers unfolds his realism. I have argued that he obtains realism too cheap because he does

\footnotetext{
${ }^{21}$ Note that evidence-transcendence is a signature of a thicker type of realism (Wright 1993: 2). That Chalmers's realism doesn't allow for evidence-transcendence shows once more that his realism is pretty thin.
} 
not address a couple of problems. A real realism cannot be developed as easily as that. The butter and the bread, it seems, is quite close to fictionalism.

All this is of course not to deny that there are cats like Tina's and that they instantiate properties in some sense. But I don't think that Chalmers's virtual realism or a different type of virtual realism is a plausible way to make sense of this. The alternative that has emerged is that we are referring to a fictional scenario, or a fiction, when we talk about Tina's cat.

Admittedly, from this perspective, virtual realism confronts us with a new interesting borderline case. Until recently, our means of fiction telling were very poor. We could talk about the fictional scenario, maybe play it on stage for two hours or produce a movie. Computers have greatly extended our ways of fiction-telling (that computer simulations have extended our capacities in other ways is stressed by Humphreys 2004). We can now produce experiences of fictional objects that do not lack behind the experiences we have from objects from the real world. Moreover, the production of the fiction is to a large extent automated. We can even allow for some interaction with the fiction. So the fiction is now so manifest that it is tempting to call it a reality of its own. I don't think we should succumb to this temptation. There isn't enough warrant for such a realism.

So, maybe, the best way to put my conclusion is to say that Chalmers's virtual realism isn't really realism, but only virtually so. ${ }^{22}$

Claus Beisbart Institute of Philosophy

University of Bern Länggassstrasse 49a

CH-3012 Bern

Switzerland Claus.Beisbart@philo.unibe.ch

\footnotetext{
${ }^{22}$ I'm grateful to an anonymous referee for detailed and constructive comments, which have led to significant improvements of this paper. Thanks also to R. Joggi for his comments. An early version of the paper was presented at KIT Karlsruhe, and I'd like to thank the audience, in particular G. Betz and M. Poznic for interesting comments and questions. Thanks also to D. Yates for his invitation to contribute this paper and for his patience.
} 


\section{References}

Barberousse, A.; Franceschelli, S.; and Imbert, C. 2009. Computer simulations as experiments. Synthese 169: 557-74.

Beisbart, C. 2018. Are computer simulations experiments? And if not, how are they related to each other? European Journal for Philosophy of Science 8: 171-204.

Beisbart, C. 2014. Are we Sims? How computer simulations represent and what this means for the simulation argument. Special issue edited by $\mathrm{P}$. Humphreys of The Monist 97(3): 399-417.

Bertschinger, E. 1998. Simulations of structure formation in the universe. Annual Review of Astronomy and Astrophysics 36: 599-654.

Bissey, F. et al. 2007. Gluon flux-tube distribution and linear confinement in baryons. Physical Review D 76: 114512.

Brass, P. 2008. Advanced Data Structures. New York: Cambridge University Press.

Chalmers., D. J. 2003. The Matrix as metaphysics. Philosophy section of the official Matrix website, also in Philosophers Explore the Matrix, ed. by C. Grau. New York: Oxford University Press, 2005: 132-76. 2 more reprints.

Chalmers, D. J. 2017. The virtual and the real. Disputatio 9(46): 309-52. Earlier draft under http://consc.net/papers/virtual.pdf

Eklund, M. 2017. Fictionalism. In The Stanford Encyclopedia of Philosophy (Winter 2017 Edition), ed. by E. N. Zalta. URL = https://plato.stanford.edu/ archives/win2017/entries/fictionalism/

Godfrey-Smith, P. 2006. The strategy of model-based science. Biology and Philosophy 21(5): 725-40.

Grüne-Yanoff, T.; and Weirich, P. 2010. The philosophy and epistemology of simulation: a review. Simulation Gaming 41: 20-50.

Hacking, I. 1983. Representing and Intervening. Introductory Topics in the Philosophy of Natural Science. Cambridge: Cambridge University Press.

Heim, M. 1998. Virtual Realism. New York: Oxford University Press.

Humphreys, P. 2004. Extending Ourselves: Computational Science, Empiricism, and Scientific Method. New York: Oxford University Press.

Kripke, S. 1980. Naming and Necessity. Cambridge (MA): Harvard University Press.

Kroon, F.; and Voltolini, P. 2017. Fiction. In The Stanford Encyclopedia of Philosophy (Winter 2016 Edition), ed. by E.N. Zalta. URL = https://plato. stanford.edu/archives/win2016/entries/fiction/

Lopes, D. M. 2001. The ontology of interactive art. Journal of Aesthetic Education 35(4): 65-81.

McDonnell, N.; and Wildman, N. Virtual reality: digital or fictional? Forthcoming in this issue.

Putnam, H. 2002. Ethics Without Ontology. Cambridge (MA): Harvard University Press

Psillos, S. 1999. Scientific Realism. How Science Tracks Truth. London: Routledge.

Quine, O. v. W. 1948. On what there is. Review of Metaphysics 2: 21-38. Reprinted in his 1953 From a Logical Point of View, Cambridge (MA): Harvard University Press, 1-19. 
Schelling, T. 1969. Models of segregation. American Economic Review 59: 488-93. Schlesinger, S. et al. 1979. Terminology for model credibility. Simulation 32: $103-4$.

Shapere, D. 1982. The concept of observation in science and philosophy. Philosophy of Science 49: 485-525.

Smuts, A. 2009. What is interactivity? Journal of Aesthetic Education 43(4): 53-73. Suárez, M. 2004. An inferential conception of scientific representation. Philosophy of Science 71: 767-79.

Walton, K. L. 1990. Mimesis as Make-believe: On the Foundations of the Representational Arts. Cambridge (MA): Harvard University Press.

Weisberg, M. 2007. Who is a modeler? British Journal for Philosophy of Science 58: 207-33.

Wildman, N.; and Woodward, R. 2018. Interactivity, fictionality, and incompleteness. In The Aesthetics of Videogames, ed. by G. Tavinor and J. Robson. London and New York: Routledge.

Winsberg, E. 2010. Science in the Age of Computer Simulations. Chicago: University of Chicago Press.

Winsberg, E. 2001. Simulations, models, and theories: complex physical systems and their representations. Philosophy of Science 68: 442-54.

Wright, C. 1993. Realism: Meaning and Truth. Collected Papers on Semantic Antirealism, $2^{\text {nd }}$ edition. Oxford: Blackwell.

Yablo, S. 2002. Go figure: a path through fictionalism. In Figurative Language, ed. by P.A. French and H. K. Wettstein. Midwest Studies in Philosophy 25: 72-102. 